\title{
Sarcoma de Kaposi Gastrointestinal primário: relato de caso
}

\author{
Primary Gastrointestinal Kaposi's Sarcoma: case report
}

Sarcoma Gastrointestinal de Kaposi primario: reporte de un caso

Yáskara Duarte Assis ${ }^{1 *}$, Larissa Lemos Pires de Melo$^{2}$, Lara Juliana Silva de Rezende², Estela Fernandes Pimenta Oliveira ${ }^{2}$, Lays Cristina Oliveira Campos², Ana Gabriela Souza Rocha², Danielle Teles de Lima², Larissa Ungarelli Borges ${ }^{2}$, Lara Braga Martins², Amanda Vilela Rodrigues.

\section{RESUMO}

Objetivo: Relatar as manifestações do Sarcoma de Kaposi em pacientes com vírus da Imunodeficiência Humana a fim de contribuir com o conhecimento teórico sobre o tema. Detalhamento do Caso: O Sarcoma de Kaposi (SK) ainda é uma malignidade comum entre pacientes com Síndrome da Imunodeficiência Adquirida, trata-se de uma neoplasia rara dos vasos linfáticos e sanguíneos, que comumente envolve a pele. As manifestações gastrointestinais, em sua maioria, têm associação com lesões cutâneas, quando isoladas ou assintomáticas são de difícil confirmação diagnóstica, o que torna a endoscopia com biópsia indispensável para o diagnóstico. O paciente em questão estava em tratamento irregular com antirretrovirais, apresentava como contagem de CD4 21 células/mcl, carga viral alta e sintomas gastrointestinais. Realizado endoscopia digestiva alta com biópsia gástrica, apresentando resultado de imuno-histoquímica positiva para SK, com expressão para CD31 e HHV-8. Considerações Finais: Conclui-se que devido ao difícil diagnóstico esse relato visa a importância da investigação de queixas gastrointestinais em paciente com HIV/AIDS, colaborando com um maior conhecimento sobre manifestações do SK nesses pacientes.

Palavras-Chave: Sarcoma de Kaposi, Síndrome da Imunodeficiência Adquirida, Neoplasia

\section{ABSTRACT}

Objective: Report the manifestations of Kaposi's Sarcoma in patients with Human Immunodeficiency Virus in order to contribute with theoretical knowledge on the topic. Details of the Case: Kaposi's Sarcoma (SK) is still a common malignancy among patients with Acquired Immunodeficiency Syndrome, it is a rare neoplasm of the lymphatic and blood vessels, which commonly involves the skin. Most gastrointestinal manifestations are associated with skin lesions, when isolated or asymptomatic, it is difficult to confirm the diagnosis, which makes endoscopy with biopsy essential for the diagnosis. The patient in question was undergoing irregular treatment with antiretrovirals, with a CD4 count of 21 cells / mcl, high viral load and gastrointestinal symptoms. Upper gastrointestinal endoscopy was performed with gastric biopsy, showing positive SK immunohistochemistry results, with expression for CD31 and HHV-8. Final Considerations: It is concluded that due to the difficult diagnosis, this report aims at the importance of investigating gastrointestinal complaints in a patient with HIV / AIDS, contributing to a greater knowledge about KS manifestations in these patients.

Keywords: Kaposi 's Sarcoma, Acquired Immunodeficiency Syndrome, Neoplasia.

\section{RESUMEN}

Objetivo: Informar las manifestaciones del Sarcoma de Kaposi en pacientes con Virus de Inmunodeficiencia Humana con el fin de aportar conocimientos teóricos sobre el tema. Detalles del Caso: El sarcoma de Kaposi (SK) sigue siendo una neoplasia común entre los pacientes con síndrome de inmunodeficiencia adquirida, es una neoplasia poco común de los vasos linfáticos y sanguíneos, que comúnmente involucra la piel. La mayoría de las manifestaciones gastrointestinales se asocian a lesiones cutáneas, cuando aisladas o asintomáticas, es difícil confirmar el diagnóstico, lo que hace que la endoscopia con biopsia sea fundamental para el diagnóstico. El paciente en cuestión estaba en tratamiento irregular con antirretrovirales, con recuento de CD4 de 21 células / mcl, alta carga viral y sintomatología gastrointestinal. Se realizó endoscopia digestiva alta con biopsia gástrica, que mostró inmunohistoquímica SK positiva, con expresión para CD31 y HHV-8. Consideraciones Finales: Se concluye que debido a la dificultad del diagnóstico, este informe apunta a la importancia de investigar las molestias gastrointestinales en un paciente con VIH / SIDA, contribuyendo a un mayor conocimiento de las manifestaciones del SK en estos pacientes.

Palabras clave: Sarcoma de Kaposi, Síndrome de Inmunodeficiencia Adquirida, Neoplasia.

\footnotetext{
${ }_{1}^{1}$ Fundação Santa Casa de Misericórdia de Franca, Franca - SP. *E-mail: yaskaraduarteassis@hotmail.com

${ }^{2}$ Faculdade de Medicina de Franca (UNIFRAN), Franca - SP.
} 


\section{INTRODUÇÃO}

O Sarcoma de Kaposi (SK) é uma patologia multifocal, definida como um tipo raro de neoplasia dos vasos linfáticos e sanguíneos a qual consiste em nódulos que frequentemente envolvem a pele, mucosas e vísceras, sendo a malignidade mais comum entre pacientes com Síndrome da Imunodeficiência Humana (AIDS) (LEE AJ, et al., 2015; CHANG Y, et al.,1994). É importante ressaltar que a taxa de SK relacionada à AIDS tem diminuído drasticamente desde a introdução da Terapia Anti-Retroviral Altamente Ativa (HAART) (BUCHACZ K, et al., 2010; BIGGAR RJ, et al., 1996).

Diferente de outras neoplasias, o SK pode não resultar de um crescimento de células tumorais de forma autonômica, mas sim em resposta a presença do Herpes vírus tipo 8 (HHV-8) ou KSHV, que tem propriedades angiogênicas em junção à uma inflamação local ou sistêmica, quando infecta células endoteliais. Ainda, além de promover a proliferação desse tipo celular, ele aumenta seu tempo de vida-média. (SCHULZ TF, CESARMAN E, 2015).

A manifestação clínica do SK pode iniciar antes mesmo do diagnóstico de AIDS, principalmente com acometimento cutâneo. Tal quadro caracteriza-se por lesões em placas violáceas e confluentes, as quais podem evoluir para cor acastanhada em diversas partes do corpo (BUCHACZ K, et al., 2010; BIGGAR RJ, et al., 1996). Pode ainda, ocorrer o acometimento do trato gastrointestinal (GI), local mais comum de acometimento visceral, além de baço, fígado ou pulmões. Porém, quando tal patologia apresenta-se de forma assintomática ou com manifestação gastrointestinal isolada torna-se um quadro de difícil diagnóstico. (MOCROFT A, et al., 2004; JOHNSON EL, et al., 2012).

É importante ressaltar a relevância da investigação de sintomas cutâneos ou viscerais e fatores de risco para SK em paciente com AIDS. Os principais fatores de risco são: CD4 <100 células / mm3, carga viral> 100.000 cópias / $\mathrm{mL}$, ausência de uso prévio de HAART, relação sexual entre homens e presença de lesões cutâneas, as quais são preditores clínicos de envolvimento gastrointestinal pelo Sarcoma de Kaposi (DEZUBE BJ, et al., 1996; NAGATA N, et al., 2012).

Com isso, é importante ressaltar que a história clínica do paciente deve ser associada aos sinais e sintomas, além de ser necessário realizar um exame complementar para confirmação diagnóstica. No caso de lesões cutâneas, recomenda-se realizar biopsia da lesão. Além disso, é importante investigar alterações em exames laboratoriais, entre eles função renal, hepática, hemograma e colher outras sorologias como hepatites e sífilis. No caso de alterações gastrointestinais, utiliza-se a endoscopia digestiva alta associada à biópsia, a qual é considerada extremamente útil para o diagnóstico de Sarcoma de Kaposi Gastrointestinal (SK-Gl) e é geralmente indicada para pacientes com sintomas de tal sistema e a presença de SK cutâneo (NASTI G, et al., 2003).

O tratamento baseia-se na Terapia Anti-Retroviral (TARV), principalmente se o paciente não realizar tratamento prévio para AIDS ou até mesmo tratamento inadequado. Além disso, em algumas situações é necessário realizar tratamento sistêmico com quimioterapia, principalmente se o paciente apresentar lesões ulceradas, doença cutânea extensa ou doença visceral, pois a patologia é rapidamente progressiva, podendo levar o paciente a óbito. Dessa forma, com tal imunodeficiência o prognóstico desses pacientes depende do início, progressão e resposta orgânica às diversas estratégias terapêuticas (ALDENHOVEN M, et al., 2006; NASTI G, et al., 2003).

O presente trabalho visa relatar as manifestações do Sarcoma de Kaposi em pacientes com vírus da Imunodeficiência Humana a fim de contribuir com o conhecimento teórico sobre o tema.

\section{DETALHAMENTO DO CASO}

Paciente, 57 anos, sexo masculino, portador do vírus da AIDS, em uso irregular da TARV (lamivudina, tenofovir, lutegravir), apresentando contagem de CD4 de 21 células/mcl e carga viral de 176.736. Relatava como antecedentes Hipertensão Arterial Sistêmica e sequela de Acidente Vascular Cerebral Isquêmico, com diminuição de força em dimídio direito. Negava tabagismo e etilismo. Fazia uso contínuo das seguintes medicações: Atenolol 50mg ao dia, Hidroclorotiazida $25 \mathrm{mg}$ ao dia, Acido acetilsalicílico $100 \mathrm{mg}$ ao dia, Sinvastatina $40 \mathrm{mg}$ ao dia, Ranitidina $150 \mathrm{mg}$ ao dia, Clonazepam $2 \mathrm{mg}$ ao dia, Azitromicina $10 \mathrm{mg}$ por semana e Bactrim diariamente. 
O paciente deu entrada em um hospital do interior do estado de São Paulo, no departamento de emergência, devido a quadro dor abdominal difusa, mais intensa em região epigástrica, associado a náuseas e vômitos, sem melhora com uso de antiemético, evoluindo com sinais de desidratação e desnutrição importantes. Além disso, apresentava um histórico de perda de 16 quilos em 6 meses, associado a sudorese noturna e mal estar generalizado.

Ao exame físico da admissão hospitalar, o paciente apresentava regular estado geral, hipocorado 2+/4+, desidratado $2+/ 4+$. Ao exame abdominal: ruídos hidroaéreos presentes em todos os quadrantes, timpanismo à percussão, dor a palpação superficial e profunda difusamente, mais intensa em região epigástrica, ausência de visceromegalias e descompressão brusca negativa. Exame físico dos aparelhos respiratório, cardiovascular e neurológico sem alterações significativas.

O paciente foi internado na enfermaria de Clínica Médica. Os exames laboratoriais de entrada evidenciavam pancitopenia (hemoglobina de $7 \mathrm{~g} / \mathrm{dL}$, hematócrito 22,1\%, hemácias 2,43 milhões, plaquetas $62.000 \mathrm{~mm}^{3}$, leucócitos de $3.800 \mathrm{~mm}^{3}$ ) e hiponatremia (sódio $115 \mathrm{mEq} / \mathrm{L}$ ), função renal e hepática sem alterações, conforme descrito abaixo (Tabela 1). Iniciado hidratação e passagem de sonda nasogástrica devido a recusa alimentar por via oral.

Tabela 1 - Descrição dos exames laboratoriais do caso relatado neste artigo.

\begin{tabular}{lcc}
\hline Exames laboratoriais & Resultados & Valores de referência \\
\hline Hemoglobina & $7 \mathrm{~g} / \mathrm{dL}$ & $13.50 \mathrm{a} 18.00 \mathrm{~g} / \mathrm{dL}$ \\
Hematócrito & $22,1 \%$ & $40.0 \mathrm{a} 54.0 \%$ \\
Hemácias & $2,43 \mathrm{milhões}$ & $4.50 \mathrm{a} 6.50 \mathrm{milhões}$ \\
Plaquetas & $62.000 \mathrm{~mm}^{3}$ & $150 \mathrm{a} 450 \mathrm{~mm}^{3}$ \\
Leucócitos & $3.800 \mathrm{~mm}^{3}$ & $5000 \mathrm{a} 10000 \mathrm{~mm}^{3}$ \\
Sódio & $115 \mathrm{mEq} / \mathrm{L}$ & $135 \mathrm{a} 145 \mathrm{mEq} / \mathrm{L}$ \\
Potássio & $4,5 \mathrm{mEq} / \mathrm{L}$ & $3,5 \mathrm{a} 5,5 \mathrm{mEq} / \mathrm{L}$ \\
Ureia & $73 \mathrm{mg} / \mathrm{dL}$ & $16 \mathrm{a} 40 \mathrm{mg} / \mathrm{dL}$ \\
Creatinina & $1,4 \mathrm{mg} / \mathrm{dL}$ & $0,6 \mathrm{a} 1,2 \mathrm{mg} / \mathrm{dL}$ \\
TGO & $30 \mathrm{U} / \mathrm{L}$ & $5 \mathrm{a} 40 \mathrm{U} / \mathrm{L}$ \\
TGP & $38 \mathrm{U} / \mathrm{L}$ & $7 \mathrm{a} 56 \mathrm{U} / \mathrm{L}$ \\
GAMA GT & $39 \mathrm{U} / \mathrm{L}$ & até $85 \mathrm{U} / \mathrm{L}$ \\
Alfa 1 glicoproteina & $104 \mathrm{mg} / \mathrm{dL}$ & 55 a $140 \mathrm{mg} / \mathrm{DL}$ \\
Ácido úrico & $4,4 \mathrm{mg} / \mathrm{dL}$ & $2,5 \mathrm{a} 7,4 \mathrm{mg} / \mathrm{dL}$ \\
TSH & $3,02 \mathrm{uUl} / \mathrm{mL}$ & $0,4 \mathrm{a} 4,5 \mathrm{uUl} / \mathrm{mL}$ \\
Chagas - IgG & Não reagente & inferior a 0,9 \\
Anti-HCV & Não reagente & inferior a 1,0 \\
VDRL & Não reagente & Inferior a $1 / 16$ \\
TAP /INR & 1,05 & 1,00 \\
\hline
\end{tabular}

Fonte: Assis YD, et al., 2020; Dados extraídos do prontuário eletrônico de um hospital do interior do estado de São Paulo.

Foram realizados os seguintes exames de imagem: ultrassonografia de abdome total, o qual evidenciou nódulos hepáticos a esclarecer, sem demais alterações; tomografia de crânio, cujo laudo relatava ausência de alterações; tomografia de tórax, a qual apresentou nódulos pulmonares sólidos e irregulares bilateralmente, além de linfonodos em mediastino; e tomografia de abdome total e pelve, com alterações em linfonodos periaórtico, caval, pélvico e inguinais.

Solicitado Endoscopia Digestiva Alta para investigação onde foram encontradas três lesões, de limites mal delimitados, mucosa arroxeada, com ulceração central e presença de múltiplas lesões ulceradas, arroxeadas, friáveis ao toque, com ulcerações em primeira e segunda porção do duodeno, sendo interrogado Linfoma de Burkitt. No momento foi realizado biópsia das lesões (Figura 1).

Após resultado do anatomo-patológico com imuno-histoquímica da biopsia gástrica (Figura 1: a), foi confirmado uma mucosa gástrica com lesão fusocelular exibindo fendas vasculares, extravasamento de hemácias e mitoses, revelando expressão para CD31 (Figura 1: b) e HHV-8 (Figura 1: c), achados consistentes com o diagnóstico de Sarcoma de Kaposi. O paciente evoluiu com piora do estado geral indo a óbito, antes do início do tratamento sistêmico com quimioterapia. 
Figura 1 - Resultado de imuno-histoquímica da biopsia gástrica do caso relatado realizada pelo Laboratório Bacchi e disponibilizada por um hospital do interior do estado de São Paulo no ano de 2019.

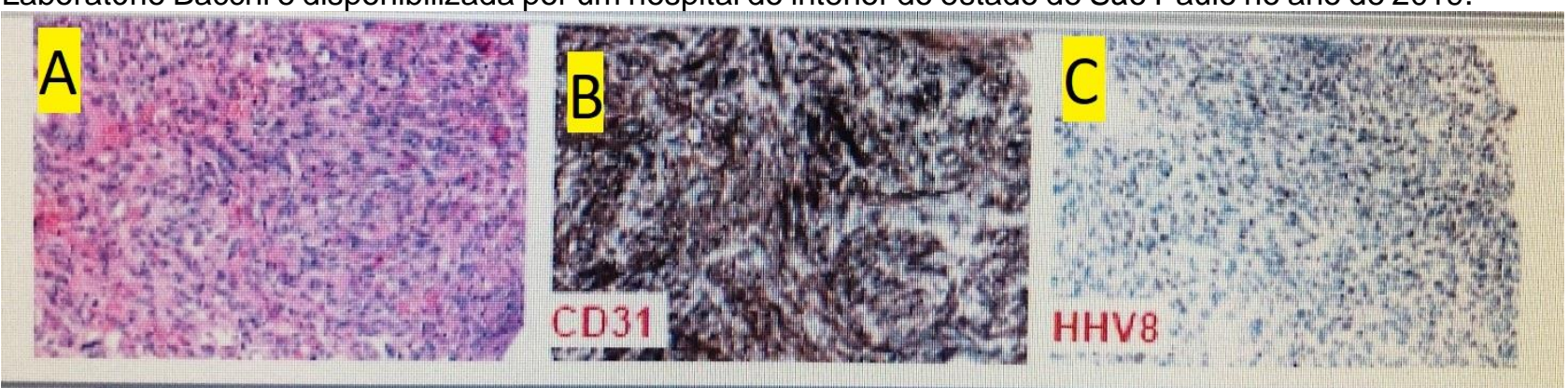

Legenda: Figura A: mucosa normal. Figura B: expressão para CD31. Figura C: expressão para HHV8. Fonte: Assis YD, et al., 2020; dados extraídos do prontuário eletrônico de um hospital do interior do estado de São Paulo.

\section{DISCUSSÃO}

O Sarcoma de Kaposi é uma patologia comum na prática médica, que por sua vez, tem apresentado redução em sua incidência nos últimos anos. Os casos em sua maioria estão associados a pacientes portadores da Síndrome da Imunodeficiência Humana (AIDS). Isto ocorre devido ao imunocomprometimento desses pacientes e propensão de alterações vasculares multifocais, juntamente a infecção pelo HH8 (LEE AJ, et al., 2015; CHANG Y, et al., 1994). Vale ressaltar que as manifestações dessa neoplasia podem ser os primeiros sinais da AIDS ou podem se desenvolver posteriormente ao diagnóstico, como no caso descrito, no qual o paciente já conhecia sua comorbidade e estava em tratamento irregular com HAART (BIGGAR RJ, et al., 1996).

No caso relatado o paciente não apresentou lesões cutâneas, manifestações comuns na abertura do quadro da neoplasia, o que levanta o questionamento acerca de um acometimento mais intenso envolvendo mucosas e TGI, característica evolucional importante para determinar o correto seguimento do quadro. (SULLIVAN RJ, et al., 2008; SOLIVETTI FM, et al., 2011).

Além da variante clínica epidêmica associada ao HIV descrita no caso existem o SK clássico, o SK endêmico em populações africanas e o SK iatrogênico, relacionado à imunossupressão. Este último, tende a ser o mais agressivo apresentando comprometimento linfonodal. $O$ subtipo endêmico tem curso mais agressivo em crianças, diferente da variação epidêmica, descrito neste relato, que acomete principalmente pacientes homossexuais, soropositivos e com idades entre 18 a 65 anos (FERNANDO ABC, et al., 2017)

Quando analisamos a origem do tumor, as teorias são controvérsias e sem comprovações, no entanto, além do uso irregular da TARV, podem estar associados a fisiopatologia os quadros infecciosos, imunológicos, endócrinos ou genéticos. Nesse sentido, o paciente pode apresentar variáveis de gravidade devido às infecções oportunistas e aos níveis de imunossupressão, o que contribui para o acometimento multifocal principalmente se não houver uso da terapia antirretroviral (COSTA DN, et al., 2008; ROHNER E, et al., 2014).

Do ponto de vista clínico, as manifestações gastrointestinais e cutâneas, associadas aos fatores de risco como carga viral $>100.000$, contribuem para investigação complementar e diagnóstico do SK. Alterações cutâneas como nódulos indolores, hiperpigmentados, de tamanhos variados, devem despertar o raciocínio médico para tal patologia (COSTA DN, et al., 2008). Nos pacientes em que tais sinais não estejam presentes, como no descrito neste relato, a suspeição pode ser dificultada, o que demostra a importância da investigação complementar frente a uma história clínica de tratamento irregular com antirretrovirais associada a sintomas como dor abdominal difusa, náuseas e vômitos, sem melhora com tratamentos usuais (SOUZA RM, et al., 2012; MOCROFT A, et al., 2004).

$\mathrm{Na}$ investigação complementar a realização da biópsia se torna essencial para descartar alguns diagnósticos diferenciais, como por exemplo, o Linfoma de Burkitt. Este, também suspeitado na EDA no caso em questão, compartilha de características clínicas do SK, podendo ocorrer em pacientes imunodeprimidos em uso irregular de antirretrovirais, com manifestações cutâneas e ou viscerais. No entanto, na imunohistoquímica as patologias se diferem, visto que os casos de Linfoma de Burkitt estão relacionados ao vírus Epstein-Baar e não ao HHV8 (DUALIBY PT, et al., 1996). 
A confirmação do diagnóstico respeita os passos descritos pela literatura, com biópsia positiva para SK na Endoscopia Digestiva Alta, com expressão para CD31 e HHV-8. Uma característica do caso descrito que o difere da maioria dos relatados, por sua vez, é o fato de, apesar de multifocal, estar restrito ao trato gastrointestinal, o que propiciaria um melhor prognóstico se o paciente houvesse realizado corretamente 0 tratamento com a HAART. Os SK em sua maioria são multicêntricos. (GRAMOLELLI S, et al., 2015; SCHULZ TF, et al., 2015).

A base fundamental para o tratamento é a terapia antirretroviral, principalmente se o paciente não realizar tratamento prévio para AIDS ou fazê-lo de forma inadequada. Seu uso representa uma redução importante na incidência de SK na população com AIDS desde que o paciente possua boa adesão ao tratamento e mantenha CD4 > 100células/mm3 e carga viral $<100.000$, diminuindo os fatores de risco para 0 desenvolvimento dessa neoplasia (NAGATA N, et al., 2012; ALDENHOVEN M, et al., 2006).

Em relação à condução médica, o SK é uma doença rapidamente progressiva, altamente invasiva e pode levar o paciente a óbito, como no caso em questão, em que as complicações das lesões intestinais culminaram em um prognóstico desfavorável. Há um consenso de que a TARV em uso regular contribui para controle da patologia e evita a extensão das lesões. Em alguns casos, os pacientes podem se beneficiar ao associar imunoterapia com interferon e até mesmo quimioterapia adjuvante, porém os estudos ainda são inconclusivos e não elucidam qual a melhor escolha para o tratamento. É importante avaliar o paciente como um todo para melhor conduta diante do quadro, individualizando a abordagem de acordo com a gravidade do caso, localização e extensão das lesões (ALDENHOVEN M, et al., 2006; NASTI G, et al., 2003; DENIS D, et al., 2016).

Conclui-se, portanto, que o caso relatado associado às referências teóricas evidencia que, mesmo com sua incidência em declínio, o SK ainda é a neoplasia mais comum entre os pacientes portadores de HIV. Sendo assim, é uma patologia que deve ser investigada em casos que apresentem fatores de riscos associados a queixas cutâneas ou gastrointestinais uma vez que o diagnóstico precoce pode mudar a evolução da doença. A endoscopia digestiva alta associada à biópsia é fundamental para investigação do Sarcoma de Kaposi Gastrointestinal (SK-GI) sendo o padrão ouro para o diagnóstico. O tratamento deve ser individualizado de acordo com a gravidade do caso, localização e extensão das lesões e baseia-se na Terapia Anti-Retroviral (TARV), podendo estar associado em algumas situações ao tratamento sistêmico com quimioterapia ou radioterapia local.

\section{AGRADECIMENTOS}

Agradecemos à Universidade de Franca (UNIFRAN) e à Santa Casa de Franca pelos dados fornecidos através do prontuário eletrônico do paciente e apoio científico, por ser ambiente de aprendizado e pelo incentivo na formação de alunos pesquisadores, permitindo a realização de atividades extracurriculares que engrandecem a formação acadêmica.

\section{REFERÊNCIAS}

1. ALDENHOVEN M, et al. Therapeutic strategies for epidemic Kaposi's sarcoma. Int J STD AIDS. 2006; 17(9): 571578.

2. BIGGAR RJ, RABKIN CS. The epidemiology of AIDS-related neoplasms. Hematology/oncology Clinics of North America, 1996; 10: 997-1010

3. BUCHACZ K, et al. AIDS-defining opportunistic illnesses in US patients, 1994-2007: A cohort study. AIDS 2010; 24(10): 1549-1559.

4. CAMPOS, FAB, et al. Sarcoma de Kaposi em Paciente Jovem Imunocompetente: uma Quinta Variante Epidemiológica? - Relato de Caso. Revista Brasileira de Cancerologia 2017; 63(4): 277-284.

5. CHANG Y, et al. Identification of herpesvirus-like DNA sequences in AIDS-associated kaposi's sarcoma. Science, 1994; 266(5192): 1865-1869.

6. COSTA DN, et al. Sarcoma de Kaposi relacionado à síndrome da imunodeficiência adquirida: características do comprometimento hepático na tomografia computadorizada e na ressonância magnética. Radiol Bras 2008;41(2):139140.

7. DENIS D, et al. First-line treatment with paclitaxel for non-HiV-related Kaposi sarcoma: experience in 10 cases. $\mathrm{Br} \mathrm{J}$ dermatol. 2016; 174(4): 905-8. 
8. DEZUBE BJ. Clinical presentation and natural history of AIDS-related kaposi's sarcoma. Hematology/oncology Clinics of North America, 1996; 10: 1023-1029.

9. DUALIBY PT, et al. Linfoma de Burkitt da órbita na AIDS Relato de um caso. Arquivo Brasileiro de Oftalmologia 1996; 59 (6): 629-631.

10. GRAMOLELLI S, SCHULZ TF. The role of Kaposi sarcoma- associated herpesvirus in the pathogenesis of Kaposi sarcoma. J Pathol. 2015; 235(2):368-80.

11. JOHNSON EL, et al. Clinical challenge: cutaneous Kaposi's sarcoma of the lower extremity. Int Wound J 2011; 8(2):163-168.

12. LEE AJ, et al. Gastrointestinal Kaposi's sarcoma: Case report and review of the literature. World J Gastrointest Pharmacol Ther. 2015; 6(3): 89-95.

13. MOCROFT A, et al. The changing pattern of kaposi sarcoma in patients with HIV, 1994-2003: The EuroSIDA study. Cancer, 2004; 100(12): 2644-2654.

14. NAGATA N, et al. Predictive Clinical Factors in the Diagnosis of Gastrointestinal Kaposi's Sarcoma and Its Endoscopic Severity. Tang J, editor. PLoS One. 2012; 7(11): e4696.

15. NASTI G, et al. AIDS related kaposi's sarcoma: Evaluation of potential new prognostic factors and assessment of the AIDS clinical trial group staging system in the haart era-the italian cooperative group on AIDS and tumors and the italian cohort of patients naive from antiretrovirals. Journal of Clinical Oncology: Official Journal of the American Society of Clinical Oncology, 2003; 21(15): 2876-2882.

16. ROHNER E, et al. Incidence rate of Kaposi sarcoma in HIV-infected patients on antiretroviral therapy in Soutern Africa: a prospective multi-cohort study. J Acquir Immune Defic Syndr. 2014 Dec;67(5):547-54.

17. SCHULZ TF, CESARMAN E. Kaposi sarcoma-associated Herpesvirus: mechanisms of oncogenesis. Curr Opin Virol. 2015; 14: 116-128.

18. SOLIVETTI FM, et al. AIDS-Kaposi Sarcoma and Classic Kaposi Sarcoma: are different ultrasound patterns related to different variants? J Exp Clin Cancer Res 2011; 30(1): 40.

19. SOUZA RM, et al. Manifestação clínica do Sarcoma de Kaposi como sinal inicial da Síndrome da Imunodeficiência Adquirida. Arq Ciênc Saúde 2012; 19(2): 30-32.

20. SULLIVAN RJ, et al. Epidemiology, pathophysiology and treatment of Kaposi sarcoma-associated herpesvirus disease: Kaposi sarcoma, primary effusion lymphoma, and multicentric Castleman disease. Clin Infect Dis 2008;47(9): $1209-1215$. 